\title{
Medication in the treatment of alcohol dependence
}

\author{
Jonathan Chick
}

Social, cultural, emotional and biological influences determine whether people drink to excess and whether they then experience harm or cause harm to others (Cook, 1994). Psychosocial treatments for alcohol dependence are only modestly successful, with most studies finding that at least $50 \%$ of patients return to harmful drinking in the following year. In the past decade there has been new evidence for the role of pharmacological treatments in reducing harm from drinking and in preventing relapse.

However, none of the treatments described here is recommended as a solo therapy. Social and psychological factors in treatment for alcohol dependence are crucial. Firstly, the chief patient variables predicting good outcome are having a job and a supportive relationship. Secondly, the most powerful therapy variable to date is therapist empathy. Finally, compliance with medication hinges on the patient's understanding and motivation, both of which are enhanced by family support and attitudes, and the therapist-patient relationship (Box 1).

\section{Assisted withdrawal}

\section{Aims}

Electively, the psychiatrist and alcohol-dependent patient may decide to facilitate commencing abstinence by reducing the short-term discomfort of withdrawal. This can be the beginning of restructuring thoughts and lifestyle towards longterm abstinence. Even in elective 'detoxification', and certainly in the non-elective situation where the drinker has suddenly been deprived of alcohol because of an accident, illness or police arrest, all care must be taken to prevent the life-threatening complications of convulsions or delirium. Anticipation is the key.

\section{When and how}

Some out-patients succeed in reducing their drinking in gradual steps. Benzodiazepines are indicated if withdrawal symptoms promise to be too uncomfortable for the patient to control the urge to seek alcohol, or there is a risk of delirium or convulsions indicated by past history, or recent consumption was more than 15 units per day for more than 10 days.

In-patient detoxification is indicated where there is a history of convulsions, incipient delirium or a living situation inimical to abstinence. When medication is not started until the day after the last drink in a severely dependent patient, as sometimes is the case in medical and surgical settings, large or frequent doses will be needed initially to titrate sedation against agitation with up to $200 \mathrm{mg}$ chlordiazepoxide or $80 \mathrm{mg}$ diazepam being required in the first 24 hours.

If the patient is vomiting, give metoclopropamide, $10 \mathrm{mg}$ intramuscularly 30 minutes before the first of the benzodiazepine tablets. Lorazepam $1 \mathrm{mg}$ is absorbed adequately from the intramuscular site, and diazepam $10 \mathrm{mg}$ can be given intravenously (Shaw, 1995).

A typical out-patient regime would be chlordiazepoxide $20-30 \mathrm{mg}$ q.i.d., or diazepam $10 \mathrm{mg}$ q.i.d. in the first 36 hours, reducing to nil over five days, giving the larger doses at night. Medication

Jonathan Chick, FRCP Ed., FRCPsych, is Consultant Psychiatrist, Alcohol Problems Clinic, Royal Edinburgh Hospital, and Senior Lecturer, Department of Psychiatry, Edinburgh University. While working for the Medical Research Council he studied the evolution of alcohol problems in distillery and brewery workers and company directors. This led to research into early detection and brief intervention for drinking problems and later to the evaluation of treatments for severely dependent drinkers. 
Box 1. Factors demonstrated to improve treatment outcome

Patient is employed and has a supportive living arrangement

Therapist empathy

Coping skills and social skills training

Supervised disulfiram

Naltrexone

Acamprosate

(Alcoholics Anonymous: not shown in controlled studies, but powerfully effective for some)

is issued on the understanding that the patient does not also take alcohol. If there is doubt that this instruction will be followed, medication is issued daily and a check made that drinking has not been resumed using an alcohol breath test, if a breathalyser is available. As required, additional doses may be needed in the first 48 hours (Table 1 ).

\section{Advice to a patient withdrawing from alcohol} at home

(1) If you have been chemically dependent on alcohol, stopping drinking causes you to get tense, edgy, perhaps shaky or sweaty, and unable to sleep. There can be vomiting or diarrhoea. This 'rebound' of the nervous system can be severe. Medication controls the symptoms while the body adjusts to being without alcohol. This usually takes three to seven days from the time of your last alcoholic drink. If you didn't take medication, the symptoms would be worst in the first 48 hours, and then gradually disappear. This is why the dose starts high and then reduces.

(2) YOU HAVE AGREED NOT TO DRINK ALCOHOL. You may get thirsty. Drink fruit juices and water but do not overdo it. You do not have to 'flush' alcohol out of the body.

Table 1. Example of dose regime for alcohol withdrawal using capsules of chlordiazepoxide $10 \mathrm{mg}$

First thing 12 noon 6 p.m. Bedtime

$\begin{array}{lllll}\text { Day 1 } & & 3 & 3 & 3 \\ \text { Day 2 } & 2 & 2 & 2 & 3 \\ \text { Day 3 } & 2 & 1 & 1 & 2 \\ \text { Day 4 } & 1 & 1 & & 2 \\ \text { Day 5 } & & 1 & & 1\end{array}$

More than three litres of fluid could be too much. Don't drink more than three cups of coffee or five cups of tea. These contain caffeine which disturbs sleep and causes nervousness.

(3) Aim to avoid stress. The important task is not to give in to the urge to take alcohol. Help yourself relax by going for a walk, listening to music or taking a bath.

(4) Sleep. You may find that even with the capsules, or as they are reduced, your sleep is disturbed. You need not worry about this lack of sleep does not seriously harm you, starting to drink again does. Your sleep pattern will return to normal in a month or so. It is better not to take sleeping pills so that your natural sleep rhythm returns. Try going to bed later. Take a bedtime snack or milky drink.

(5) The capsules may make you drowsy so you must not drive or operate machinery. If you get drowsy, miss out a dose.

(6) Meals. Even when you are not hungry, try to eat something. Your appetite will return.

\section{Complications}

\section{Wernicke-Korsakoff syndrome}

Wernicke-Korsakoff syndrome seems often to be precipitated by hospital admission and withdrawal from alcohol. The physiological stress of withdrawal may contribute; as may resuming intake of carbohydrates, breakdown of which requires enzymes dependent on thiamine and uses up remaining stores of that essential component of neuronal metabolism. It is good preventive practice to prescribe thiamine $200 \mathrm{mg}$ orally daily during the withdrawal phase. If the patient is ataxic or obviously malnourished, give thiamine parenterally. The currently available vitamin B injection, 'Pabrinex', has not acquired a reputation for allergic reactions, but administration should be in a setting where resuscitation facilities are available in case of anaphylactic shock. Anaphylaxis is less likely with intramuscular than intravenous injection, and of the intravenous routes perhaps slow infusion saline drip is preferable to slow bolus injection.

Treating Wernicke-Korsakoff syndrome. If confusion, incontinence, ataxia or strabismus are noted in a patient withdrawing from alcohol, intravenous thiamine must be given immediately and hypoglycaemia considered. Then, consideration may given to other potential causes, such as subdural haematoma or hepatic encephalopathy. 


\section{Convulsions}

Deaths have occurred in hospital, prison and police cells from bursts of alcohol withdrawal fits. Elective withdrawal from alcohol in patients with a history of fits of any cause can be made safer by commencing an anticonvulsant (e.g. phenytoin or carbamazepine) four days before cessation of drinking. This permits a therapeutic serum level to be achieved in good time. Alternatively, larger than normal doses of long-acting benzodiazepines are given in the first 36 hours, and should be started not after the blood alcohol level has fallen to zero but before. If the patient is sober enough to cooperate appropriately with admission, the psychiatrist should commence benzodiazepines while the patient still smells of alcohol.

A patient died at 6 p.m. in a convulsion having been admitted at 12 noon to a psychiatric ward. The nurses, adhering to the prescribed 6 hourly regime, had waited until 6 p.m. to give him his first dose of sedative.

Treating convulsions. With the aim of preventing further convulsions, the patient is given $10 \mathrm{mg}$ diazepam intravenously or rectally. Give double the dose in a patient who has been taking benzodiazepines regularly prior to this event, or is much above average weight. A convulsion may presage a severe withdrawal syndrome, and parenteral thiamine should be given. It is illogical to commence an anticonvulsant which may take 2-3 days to reach a therapeutic serum level. Rather, increase the benzodiazepines, which are effective in controlling alcohol withdrawal fits.

\section{Delirium tremens}

If confusion and hallucinations develop, this is often 48-72 hours after the last drink. Sufficient benzodiazepine, given soon enough in the withdrawal phase, reduces the risk. Good nursing in a welllit, calm environment is preventive. Explaining things and orientating the patient reduces anxiety, paranoia and confusion.

Treating delirium tremens. Increasing the dose of the benzodiazepine may be sufficient. If not, the slight epileptogenic effect of phenothiazines should not deter the psychiatrist from prescribing these to control delusions and hallucinations, if anticonvulsant protection by a benzodiazepine is in place. The right environment and nursing help greatly in reducing the risk of aggression.

\section{Alcoholic hallucinosis}

Hallucinations, perhaps with secondary delusional beliefs, may develop during a period of heavy drinking or of relative reduction in drinking, or may be found to persist after the acute symptoms of delirium tremens resolve. Abstinence plus chlorpromazine (e.g. $50-100 \mathrm{mg}$ b.d.) or other major tranquilliser usually results in complete recovery over the coming months, permitting withdrawal of the medication. However, a proportion of such illnesses persist and are later diagnosed as schizophrenia or affective psychosis (Cutting, 1978).

\section{Which sedative?}

The longer-acting benzodiazepines, diazepam and chlordiazepoxide, are the most successful in reducing anxiety and the risk of convulsions. Both have active metabolites, which themselves require excretion by the liver and so may cause a cumulative over-sedation in the elderly or those with liver failure, unless progressive reduction is made appropriately. Lorazepam and oxazepam have intermediate half-lives and do not produce active metabolites, being inactivated and eliminated by simple glucuronidation. Dosage may be harder to titrate than for diazepam or chlordiazepoxide.

Withdrawal symptoms can be controlled with chlormethiazole. It has the disadvantage that it is relatively short-acting (elimination half-life 3-6 hours). It should not be given to out-patients because of the risk of respiratory depression, if taken in overdose or with large amounts of alcohol. It has addictive properties, perhaps because of its rapid action and short half-life, which has led to patients seeking prescriptions after detoxification who develop dependence with subsequent withdrawal convulsions. Intravenous infusions, although effective in emergency situations such as delirium tremens in a postoperative patient, have caused fatal respiratory depression, and should be used only where intubation and ventilation facilities are on hand, and with great care in chronic pulmonary insufficiency and advanced liver disease.

\section{Deterrent medication to reduce relapse}

\section{Disulfiram}

\section{Action}

Disulfiram, if taken regularly in a sufficient dose, causes an unpleasant reaction 15-20 minutes after alcohol enters the body. The reaction is due to accumulation of the intermediate metabolite of ethanol, acetaldehyde. The patient flushes, 
Box 2. Procedures for successful use of deterrent drugs

Physical examination, cardiac history and baseline liver function tests.

Explain actions of the drug.

Negotiation proceeds so that the patient not only accepts the drug but wants it.

Information leaflet for patient and relatives.

Offer psychosocial support, and help to cope with thoughts of drinking and handling previous triggers, e.g. out-patient group, AA.

Facilitate the patient inviting a third party 'to help him/her remember to take the drug', e.g. partner, family member, clinic staff, nurse or supervisor at work.

Set up acceptable supervision regime tablet(s) seen to have the correct markings (i.e. not soluble aspirin) are dispersed in half glass of water and taken in view of supervisor; frequency agreed (e.g. daily or thrice weekly).

Agreement that supervisor phones clinician if patient appears to be changing the plan; and that clinician phones patient to ask reasons for change of plan.

When supervision is at the clinic: properly briefed staff; delays avoided; approach/ atmosphere welcoming and reinforcing.

Liver function tests repeated once after 1-2 months.

experiences headache, pounding in the chest or head, tightness in breathing, nausea and perhaps vomiting. Unwanted effects of the drug itself are few, with drowsiness or headache the only common ones. A taste in the mouth, or an odour on the breath, are sometimes experienced. Loss of libido is attributed by some patients to disulfiram, but does not occur more frequently than in placebotreated control patients.

The liver acetaldehyde dehydrogenase isoform that disulfiram blocks seems to have a turnover of several days. The alcohol-disulfiram reaction is usually only experienced in patients who have taken it for 3-4 days. A loading dose is recommended. The enzyme appears to stay blocked for several days, with alcohol-disulfiram reactions occurring up to 7 days after the last dose.

It is recognised practice to increase the dose of disulfiram to 300 or $400 \mathrm{mg} /$ day, if the patient has taken alcohol and the reaction has not been severe enough to act as a deterrent. A few tolerate the reaction, do not wish to increase the dose, and use the drug as a way of controlling their drinking rather than abstaining (in which case the clinician records that the patient does this at his own wish and against advice).

\section{Mode of use (Box 2)}

Disulfiram is an aid. It enables the individual to get used to life without alcohol and allows time for confidence to resume in the family and at work. It acts as a deterrent to temptation and thus helps postpone further alcohol crises. Damaged organs have time to recuperate. Patients suspended because of drinking might be reinstated if the employer knows disulfiram is being used.

If drowsiness is a side-effect, it is taken at bedtime. It is common to prescribe it for six months, but many patients want to continue the method longer. Alternatively, patients may keep a supply to use when they feel they are going to be at risk of drinking; for example, a business trip away or a social event.

Patients may object that it is a sign of weakness to be taking disulfiram, instead of using will-power. Unfortunately, will-power is not always there when most needed, so disulfiram acts as a last line of defence. With the pills, a decision whether to drink or not still has to be made, but only once a day.

Tablets implanted subcutaneously sensitise the patient for about two weeks only, not for months. This, together with the risk of local irritation, has led to the virtual disappearance of this approach.

\section{Risks}

The dangerous component of the disulfiramethanol reaction is the hypotension or cardiac disturbance. Disulfiram is contraindicated after myocardial infarction, angina or arrhythmia. If such patients strongly request to use disulfiram, perhaps because they know that a bout of drinking could be as dangerous as an alcohol-disulfiram reaction, a statement of the added danger should be given and acknowledged by the patient. Many doctors prescribe disulfiram without a screening electrocardiogram, but the medical history and pulse must be checked. For many years now, since doses of $500 \mathrm{mg}$ or more have ceased to be used, there have been no deaths due to the disulfiramethanol reaction, either reported in the literature, or in areas of frequent prescribing such as Scandinavia.

Disulfiram can provoke a psychotic state in a predisposed individual and should not be given to someone with a history of paranoid thinking or psychotic illness.

Peripheral neuropathy is an infrequent toxic effect in patients who have used disulfiram in high 
doses (usually $\geq 400 \mathrm{mg}$ ) for many months and may be more common in association with tricyclics. It is reversible, but contraindicates re-exposure to the compound.

\section{Drug interactions}

Serum levels of anticonvulsants and tricyclics may be enhanced in patients taking disulfiram. The metabolism of caffeine, warfarin, chlordiazepoxide and diazepam is delayed by disulfiram. It should not be given with metronidazole, since the combination has caused a confusional state.

\section{Supervision}

Disulfiram works only if taken consistently. The randomised controlled studies that have demonstrated efficacy (and shown near 100\% two-year abstinence when combined with contingent marital and community rewards) have entailed recruiting a supervisor. Patients are encouraged to ask their partner, a nurse or welfare officer at work, or a nurse at the health centre or the clinic to see them take the disulfiram. This can either be on a daily basis, or three times a week as long as the total number taken per week is at least seven $200 \mathrm{mg}$ tablets. The product is now sold in a dispersible form to take in water so that it can be seen to be swallowed. Most find it tasteless (Box 3).

\section{Disulfiram and the liver}

There have been rare reports of hepatitis in patients newly starting disulfiram, mostly in women. Because of this, some doctors recommend that regular liver function tests are performed. However, since the reactions reported are in the early weeks of therapy, a check after one month rather than repeated tests is probably all that is necessary. A randomised trial of disulfiram versus vitamin C as control found those on disulfiram showed greater improvement in mean serum gamma glutamyl transferase than controls (Chick et al, 1992). The disulfiram group drank less, which explains why their mean gamma glutamyl transferase improved more, but it substantiated other work which had failed to show that disulfiram impaired liver function.

Because hypersensitivity could be fatal in someone with a compromised liver, great caution should be used in patients with advanced liver disease and a rule of thumb might be not to prescribe it when the serum bilirubin is $>25 \mathrm{mmol} / 1$ and the serum albumin is below normal, low serum albumin being an indication of liver failure. Also, disulfiram is metabolised hepatically and might strain a diseased liver. However, elevated liver
Box 3. Deterrent medication: information for patient and partner

A partner is a person who is asked by the patient to observe the taking of the Antabuse tablets.

So that other tablets cannot be substituted, the genuine Antabuse tablets are marked Dumex $110 \mathrm{~L}$ (Dumex is the manufacturer).

To ensure that they are not placed under the tongue and removed later, Antabuse tablets should be dissolved in half a glass of water (the tablets break up and disperse and the mixture is tasteless).

It does not matter what time of the day the tablet is given. If it is more convenient, it can be given on three days a week (i.e. instead of one tablet daily it can be taken two on Monday, two on Wednesday and three on Friday, for example).

If it is suspected that the patient has decided to vomit after taking the tablet, the partner can stay with the patient for up to 30 minutes after the tablet is taken (this is rarely necessary).

If the patient decides to stop taking the tablets, the patient or the partner should telephone the treating doctor or a member of nursing staff so that the reason for this may be discussed.

enzymes, which are found in $60-70 \%$ of patients with alcohol problems in psychiatric practice in the $\mathrm{UK}$, are not a contraindication to disulfiram.

\section{Calcium carbimide}

If disulfiram cannot be prescribed because of contraindications or unwanted effects, calcium carbimide is available. In the UK its license is not currently retained, apparently for commercial rather than safety reasons. It has the proprietary name 'Abstem' in the UK ('Temposil' in Canada) and is prescribed on the basis of "named patient, physician's responsibility", that is, the manufacturer (Cyanamid) does not offer indemnity.

Calcium carbimide may block a different isoform of liver acetaldehyde dehydrogenase than disulfiram - the alcohol reaction can occur any time up to 36 hours after a dose, which is much shorter than the duration of action of disulfiram. The dose of calcium carbimide is $100 \mathrm{mg} /$ day. Thyroid depres- 
sion and reduced white cell count have been reported.

\section{Motivating patients to use a deterrent}

Many patients who decide the advantages of stopping drinking outweigh the advantages of carrying on, will accept a deterrent. If rewards of abstinence continue, they will persevere. They may be glad to find the craving for alcohol wanes: "I know I dare not drink, so the debate in my head whether or not to drink evaporates."

Many, however, cease taking the tablets after two or three weeks, especially if there is no supervision arrangement. Benefits in the marriage are a powerful reinforcer. The therapist can facilitate this by:

(1) improving communication;

(2) helping each partner to express their needs with assertion not aggression; and

(3) instructing each partner to reward and encourage positive behaviours in the other.

Keeping their job and getting rewards at work will boost motivation. For those unemployed and living alone, the rewards of feeling more competent and autonomous will help, as will membership of groups such as Alcoholics Anonymous (AA).

Some repeat offenders ask for deterrent drugs because they recognise that relapse coincides with re-offending. Psychiatrists reporting to a court can offer deterrent drugs to repeat offenders as a part of a treatment package. Sometimes, courts wishing to assist rehabilitation or to avoid a custodial sentence, defer sentence and ask the psychiatrist to report in three or six months on whether the offender has responded to treatment. This invokes an element of coercion in the treatment. However, just as a spouse's threat to leave, or pending dismissal from work, often correlate with a period of renewed effort to stop drinking, so also the offender may assiduously follow a treatment plan if he has to return to court in a few months. This is slightly different from a court mandating probation with disulfiram as a condition, which puts the psychiatrist in a different relationship to his patient, and one which many would find uncomfortable.

\section{Treatment duration}

Initially, suggest at least six months. There is a gradual drop-out over the weeks, as some patients decide to resume drinking and stop the medication. The supervision arrangement may have become lax. However, there are patients, and families, who ask to continue the deterrent method for longer. There is no evidence of long-term toxicity in patients taking $200 \mathrm{mg}$ disulfiram daily, though there has been a report of cognitive impairment (reversible) in a patient taking a larger dose for many years (Borrett et al, 1985). There are currently many around the world who have used disulfiram for years.

\section{New advances}

There are genetic as well as environmental contributions to why alcohol problems run in families. This has spurred on research into the neurobiology of addictions (see Nutt, 1996). There are strains of laboratory animals predisposed to take alcohol that, given the opportunity, work for alcohol and show withdrawal symptoms.

The neurotransmitters involved in reward, repetitive behaviours and drug-seeking behaviours include endorphins, dopamine and serotonin. The $\gamma$-aminobutyric acid (GABA)/glutamate systems are important.

Ethanol substantially reduces activity of voltagegated calcium channels at the nerve cell membrane and interferes with the $N$-methyl-D-aspartate (NMDA) control, which leads to depression of the excitatory glutamate system. Animals dependent on ethanol, but no longer exposed to it, are found to have increased glutamate activity because of compensatory overactivity of calcium channels and reduced NMDA control.

The two drugs below have been tested in randomised controlled trials and shown modest but clinically useful efficacy.

\section{Acamprosate (calcium acetyl homotaurinate)}

This enhances GABA transmission and antagonises glutamate transmission, without any benzodiazepine-like anxiolytic action, probably by affecting calcium channels and NMDA receptors. It reduces drinking in alcohol-dependent animals, and reduces the reinstatement of drinking behaviour and withdrawal symptoms in animals reexposed to alcohol after a period of abstinence. It does not substitute for ethanol or benzodiazepines in such animals in the sense that they will seek out acamprosate (reviewed by Littleton, 1995). It has a dose-related effect in improving abstinence rates in recently detoxified patients (Paille et al, 1995). Other impressively large randomised controlled studies of acamprosate have shown an effect, typically of 
enhancing complete abstinence by some $20 \%$ for up to one year (e.g. Whitworth et al, 1996; Sass et al, 1996). Other studies have been published but differ in the extent to which sedatives were permitted in the first weeks and in the outcome criteria (Chick, 1995). Acamprosate is available on prescription in the UK and most European countries.

\section{Naltrexone/nalmefene}

These antagonise the brain's endogenous opiate transmitters, endorphins, which are released as one of many acute actions of ethanol on the limbic system. Individuals with a high genetic loading for alcohol dependence may inherit an oversensitive endorphin release after ethanol, which might contribute to the loss of control experienced by some drinkers (Gianoulakis et al, 1996). Naltrexone reduces ethanolseeking in dependent animals.

Two double-blind, randomised controlled studies of naltrexone in detoxified patients taking part in an out-patient treatment programme have been published and show a reduced risk of relapse, at least for three months. The same appears to be true for nalmefene (Mason et al, 1994). The effect size of naltrexone treatment in reducing the percentage of days drinking was 0.42 in one study and 0.60 in the other (reviewed in Volpicelli et al, 1995). For comparison, the mean effect size in meta-analyses of other studies of fluoxetine in the treatment of depression is around 0.4 (Greenberg et al, 1994). Results of longer studies, and larger samples, are awaited. In 1995 naltrexone was licensed in the United States for use as part of a comprehensive treatment programme for alcohol dependence, and in Canada and Austria the drug was licensed in 1996. At the time of writing, naltrexone is licensed in the UK for opiate addiction (because it removes the euphoria of taking heroin) but not in alcohol dependence, for which indication it is currently prescribed on the principle: "named patient, physician's own responsibility".

Some patients who resume drinking while taking naltrexone report that they feel less of the ethanol 'high'. Perhaps they then experience less impulse to carry on drinking (Volpicelli, 1995; Volpicelli et al, 1995). However, there is an increase in the number of patients who report achieving total abstinence as well as a reduction in drinking overall. Early speculation that opiate antagonists might cause dysphoria seemed to be supported by statements from heroin addicts given naltrexone to help them abstain from opiates. However, laboratory studies and randomised controlled trials have not found consistent evidence of dysphoria or loss of feelings of pleasure in either normal volunteers or alcoholics (for example, see Doty \& de Wit, 1995).

\section{Action and use of the new agents}

It is possible that the reduced likelihood of picking up the first drink, and the reduced craving for alcohol shown in some studies, is because the strength of the previous triggers - emotional, cognitive or environmental - is attentuated by the drugs' actions in certain pathways in the limbic system. In time these will be elucidated. More information is now needed on which patients respond, what is the optimal timing and duration of use, and whether, and if so which, psychological and social interventions are necessary complements. Currently, it is reommended that the drugs are prescribed at the beginning or soon after commencement of detoxification. Naltrexone is given as one tablet once daily, and acamprosate three times daily. Neither drug is addictive in the sense that there is a withdrawal syndrome. Follow-up has not revealed rapid relapse after cessation of these drugs. Both have a good safety record and it is notable that acamprosate is not metabolised in the liver. Neither drug exacerbates psychomotor impairment caused by alcohol. It is likely that poor compliance, unless resolved, will limit the effectiveness of these new products, although perhaps less than it has with the deterrent drugs.

Since they are expensive relative to disulfiram, and of course to AA (which costs the National Health Service nothing), the use of naltrexone and acamprosate may initially be cautious. Perhaps they should be used only in patients who have failed to respond to brief psychological intervention. Such patients would at least see the logic of using an aid to abstinence and be more likely to comply with medication. However, the costs to individuals, families, the NHS and society of persistent, relapsing alcohol dependence should also be weighed.

\section{Treatment of coexisting affective disorder}

\section{Depression}

Depression is common in patients dependent on alcohol. It may be a result of drinking, or loss of friends, family or work, with resulting feelings of hopelessness, guilt and lack of direction. They may have little appetite because they are drinking instead, and may have lost energy and sexual drive because of lowered serum testosterone. They may wake in the small hours of the night feeling anxious because of the rebound wakefulness of alcohol 
withdrawal. Those signs and symptoms of depressive illness commonly clear with abstinence, help in tackling or tolerating the problems that exist, and getting relationships on to a better footing

However, in some patients (women more than men) a depressive episode preceded the alcohol dependence. Alcohol was taken in part as selfmedication. Or, despite abstinence, depressive symptoms are found to continue. In this case, antidepressants should be offered in the usual way. Relapsing alcoholism, if secondary to depressive illness, is an indication for long-term antidepressants. Lithium is not a treatment for alcohol dependence itself, but is effective if it is secondary to manic-depressive disorder.

\section{Anxiety and panic disorder}

A patient may have had panic attacks for years before discovering that alcohol could end or prevent an attack. Other patients may have had the first panic attack due to alcohol withdrawal, but the attacks then continued, even into sustained periods of abstinence. In either case, when cognitive-behavioural therapy is not sufficient, medication is indicated, especially if growing physical or social damage is accruing at every alcoholic relapse.

Benzodiazepines are immensely helpful to such patients. However, their use should normally be only short-term because there is a risk that tolerance will develop with aggravation of symptoms due to increasing partial withdrawal at periods between doses. Buspirone has been studied in such patients. One study (Kranzler et al, 1994) found an advantage in terms of drinking and anxiety but an earlier study (Malcolm et al, 1992) did not.

Tricyclic antidepressants and SSRIs, such as paroxetine, have a role in panic disorder. However, those anxious patients who have become dependent on alcohol seem to experience a high rate of unwanted effects. With SSRIs, even titrating up from the lowest dose possible, they may need an anti-emetic such as metoclopropamide, $10 \mathrm{mg}$ six hourly (maximum $30 \mathrm{mg}$ in 24 hours) for a few days, and/or a reducing benzodiazepine regime for three weeks, if they are to be persuaded to give the drug a chance to show its effect.

There are some patients with long histories of alcohol dependence, who have failed to respond to numerous psychological and other treatment ventures, and who have pronounced panic disorder. Unless the patient is already a purchaser of street drugs, the risk of complications from repeated prescribing of a long-acting benzodiazepine are so much less than the risks from alcohol excess that it can sometimes be humane to the individual and the family to prescribe the benzodiazepine. If prescribed (and to do so is controversial), it should be dispensed in limited aliquots or issued daily by a family member. It should be conditional on abstinence from alcohol, which can be aided by disulfiram if necessary. The patient should usually be encouraged to use an antidepressant too.

'As required' use, for example, for travelling on public transport, is to be preferred to regular use in order to limit the development of tolerance, even though such use perhaps perpetuates the underlying phobic beliefs.

\section{Reducing harm in patients who cannot or will not stop drinking heavily}

There is research into how, pharmacologically, liver cirrhosis might be prevented in those who carry on drinking. Propylthiouracil has been used to limit the progression of alcoholic cirrhosis once diagniosed.

There has never been a controlled study to show that regular thiamine supplements in committed drinkers can prevent the development of WernickeKorsakoff syndrome or, indeed, the risk of alcoholic neuropathy or dementia. Nevertheless, it seems prudent that individuals who drink heavily enough to reduce their absorption of thiamine, or seldom eat, or have a family history of Wernicke-Korsakoff syndrome, should be encouraged to take B-vitamin supplements.

\section{References}

Borrett, D., Ashby, P., Bilbao, J., et al (1985) Reversible late onset disulfiram-induced neuropathy and encephalopathy. Annals of Neurology, 17, 396-399.

Chick, J. (1995) Acamprosate as an aid in the treatment of alcoholism. Alcohol and Alcoholism, 30, 785-787.

-, Gough, K., Falkowski, W., et al (1992) Disulfiram treatment of alcoholism. British Journal of Psychiatry, 161, 84-89.

Cook, C. (1994) Aetiology of alcohol misuse. In Seminars in Alcohol and Drug Misuse (eds J. Chick \& R. Cantwell), pp. 94 125. London: Gaskell.

Cutting, J. (1978) A re-appraisal of alcoholic psychoses. Psychological Medicine, 8, 285-295.

Doty, P. \& de Wit, H. (1995) Effects of naltrexone pretreatment on the subjective and performance effects of ethanol in social drinkers. Behavioural Pharmacology, 6, 386-394.

Gianoulakis, C., Krishnan, B. \& Thavundayil, J. (1996) Enhanced sensitivity of pituitary $\beta$-endorphin to ethanol in subjects at high risk of alcoholism. Archives of General Psychiatry, 53, 250-257.

Greenberg, R. P., Bornstein, R. F., Zborowski, M. J., et al (1994) A meta-analysis of fluoxetine outcome in the treatment of depression. Journal of Nervous and Mental Diseases, 182, 547551. 
Kranzler, H. R., Burleson, J.A., Boca, F. K., et al (1994) Buspirone treatment of anxious alcoholics. Archives of General Psychiatry, $51,720-731$.

Littleton, J. (1995) Acamprosate in alcohol dependence: how does it work? Addiction, 90, 1179-1188.

Malcolm, R., Anton, R. F., Randall, C. L., et al (1992) A placebocontrolled study of busprione in anxious in-patient alcoholics. Alcoholism: Clinical and Experimental Research, 16, 1007-1013.

Mason, B. J., Ritro, F. C., Morgan, R. O., et al (1994) A doubleblind placebo-controlled study to evaluate the efficacy and safety of oral nalmefene $\mathrm{HCl}$ for alcohol dependence. Alcoholism: Clinical and Experimental Research,18, 1162-1167.

Nutt, D. (1996) Addiction: brain mechanisms and their treatment implications. Lancet, 347, 31-36.

Paille, F. M., Guelfi, J. D., Perkins, A.C., et al (1995) Randomised multicentre trial of acamprosate in a maintenance programme of abstinence after alcohol detoxification. Alcohol and Alcoholism, 30, 239-247.

Sass, H., Soyka, M., Mann, K., et al (1996) Relapse prevention by acamprosate: results from a placebo controlled study in alcohol dependence. Archives of General Psychiatry, 53, 673680

Shaw, G. K. (1995) Detoxification: the use of benzodiazepines. Alcohol and Alcoholism, 30, 765-770.

Volpicelli, J. R. (1995) Naltrexone in alcohol dependence. Lancet, 346, 456.

- Volpicelli, L. A. \& O'Brien, C. P. (1995) Medical management of alcohol dependence: clinical use and limitations of naltrexone treatment. Alcohol and Alcoholism, 30, 789-798.

Whitworth, A. B., Fischer, F., Lesch, O., et al (1996) Comparison of acamprosate and placebo in long-term treatment of alcohol dependence. Lancet, 347, 1438-1442.

\section{Multiple choice questions}

\section{Disulfiram:}

a is contraindicated in patients with raised serum gamma glutamyl transferase

b causes tiredness, an unwanted effect

c should be avoided in patients referred by the courts

d if supervised by the spouse, can improve outcome

e can be taken as a tablet dispersed in water.

2. Panic disorder in alcohol dependence:

a may have been caused by alcohol excess

b is associated with sensitivity to the side- effects of SSRIs

c is a complete contraindication to long-term benzodiazepine prescription

d can be helped by tricyclic antidepressants

$e$ is a cause of repeated relapse into problematic drinking.

3. In alcohol withdrawal:

a convulsions can be fatal

b fits can be best treated by immediate prescription of phenytoin

c confusion and hallucinations may take 72 hours to manifest

d phenothiazines are contraindicated if delirium tremens develops

e sedatives can be started before the blood alcohol has fallen to zero.

4. It is true of Wernicke-Korsakoff syndrome that:

a patients are seldom under the age of 50

b malabsorption of thiamine contributes

c it is avoided in developed countries because alcoholics are sufficiently nourished

d intake of carbohydrate may precipitate symptoms

e it can be prevented by giving prophylactic parenteral vitamins in the poorly nourished patient undergoing alcohol withdrawal.

\begin{tabular}{|c|c|c|c|c|}
\hline \multicolumn{5}{|c|}{ MCQ answers } \\
\hline 1 & 2 & & 3 & 4 \\
\hline a $F$ & $\mathbf{a}$ & $\mathbf{T}$ & a $\mathrm{T}$ & a $F$ \\
\hline b $\mathrm{T}$ & b & $\mathrm{T}$ & b F & b $\mathrm{T}$ \\
\hline c $\mathrm{F}$ & c & $\mathbf{F}$ & c $\mathrm{T}$ & c $F$ \\
\hline d $\mathrm{T}$ & d & $T$ & d F & d $\mathrm{T}$ \\
\hline e $\mathrm{T}$ & e & $\mathrm{T}$ & e $\mathrm{T}$ & e $\mathrm{T}$ \\
\hline
\end{tabular}

